\title{
Family medicine into the future: blending health and cultures
}

\section{WONCA EUROPE 2010 CONFERENCE REPORT}

The 16th WONCA Europe conference began with excitement, and it certainly did not disappoint. As 2800 GPs gathered in a packed auditorium on the outskirts of Malaga, what better way to start than with a fiery flamenco performance? The anticipation was palpable, as we were treated to a passionate performance of guitarra, cante y baile (flamenco guitar, singing and dance). After this dizzying start, the next major challenge was deciding which of the 306 oral presentations and 57 workshops to attend, not to mention six keynote lectures and 740 posters from Belgian to Brazilian authors.

As young GPs we were privileged to be welcomed into the Vasco da Gama Movement (VdGM) community, WONCA Europe's network for trainees and newly qualified GPs. This opened up opportunities to share ideas, collaborate on international projects, renew our passion for family medicine and above all, make friends and have fun!

One example of the cross-national collaboration that the VdGM has enabled is the successful implementation of a panEuropean questionnaire. Based on the European definition of general practice, on which the RCGP curriculum is also based, this study looks into motivation and satisfaction with GP training. VdGM presented preliminary results from over 3700 GP trainees in seven European countries. These provided a fascinating snapshot into differences in working hours, income and satisfaction for GP trainees in a range of European countries. Further analysis of these findings will be fed back into national curriculum development boards of participating countries in order to work together towards improving the quality of GP training across Europe.

The VdGM and European GP Research Network (EGPRN) research methods workshop was filled with delegates from a range of backgrounds, from trainees through to experienced GPs who were looking to embark on research for the first time. The room was a hive of lively discussions, as participants set to work devising their potential methods to answer questions such as 'How common is deafness and ear disease in Nepal?'. It quickly became apparent how there may be a multitude of different ways to do research which can be equally appropriate, as well as the vast array of opportunities that research can bring to one's career.

The differences in GP training across the European Union were highlighted in the joint VdGM and European Academy of Teachers in General Practice and Family Medicine (EURACT) workshop. Questions were raised such as how long should GP training be, how much of this time should be spent in general practice, and how is knowledge assessed? The significant differences across Europe became glaringly evident as discussions progressed. In some European countries you can practice as a GP straight out of medical school, in others there is vocational training, however, this may be spent entirely in hospital posts or there may not be an exit exam. This raises the question of whether the EU is really ready for the free movement of doctors in the labour market or is patient safety being compromised? It also highlighted the value of WONCA to draw together professionals to work together towards improving the standards of training.

Interest in VdGM Hippokrates exchange programme continues to expand. Trainees sat on the floor and stood in the corridor when there was no more room left in the VdGM exchange workshop for participants. The workshop heard British and Spanish experiences of being a visitor and host respectively. It also heard the Dutch trainee international committee's experience of running an international group exchange during a national Dutch GP conference.

Across the 3 days of the conference the keynote lectures explored a number of topical themes. Highlights included Professor Linn Getz (Iceland) who drew attention to a growing field of evidence in neuro-endocrinology, psycho-neuroimmunology, neuro-imaging and epidemiology that demonstrates how personal experiences are closely intertwined with biological responses; thus the importance of carefully listening to the patient's narrative. The final keynote lecture by Dr Yvonne van Leeuwen and $\mathrm{Dr}$ Erik Teunissen (Netherlands) addressed the future generations of general practice. A map of Europe was peppered with examples, among them the UK's First5 initiative spearheaded by Dr Clare Taylor, demonstrating how young GPs had collaboratively led change and innovation at national and international levels.

There were many highlights; the main one being the opportunity to meet and share experiences with family doctors from around the 65 different countries represented. As well as the busy conference schedule there was time for those crucial conversations over tapas that cemented friendships with newly-met colleagues. The Dutch trainees hosted their popular annual party while the closing cocktail party was held in the fabulous Malaga automobile museum.

Despite the challenges and changing times we are facing as young doctors in general practice, seeing the wider picture enabled us to put things into perspective and we came away with renewed energy; feeling that overall, the future of general practice is looking bright!

\section{Elizabeth Brown, Madeleine Attridge, Luisa Pettigrew, and Jessica Watson}

WONCA World. www.globalfamilydoctor.com WONCA Europe. www.woncaeurope.org Vasco da Gama Movement. www.vdgm.eu

WONCA Europe Conference 2011. 8-11 September 2011, Warsaw. www.woncaeurope2011.org

DOI: 10.3399/bjgp11X556948 